\title{
Convergence of Optimization Problems
}

\author{
K. Jeyalakshmi
}

\begin{abstract}
In this paper we consider a general optimization problem (OP) and study the convergence and approximation of optimal values and optimal solutions to changes in the cost function and the set of feasible solutions. We consider the convergence optimization problems under the familiar notion of uniform convergence. We do not assume the convexity of the functions involved. Instead we consider a class of functions whose directional derivatives are convex. They are known as locally convex functions or following Craven and Mond nearly convex functions. We given necessary preliminaries and we prove that a sequence of locally convex optimization problems converge to a locally convex problem. We also prove that uniform convergence of locally convex optimization problems implies epi-graph convergence of the problems. Even though for simplicity we have taken locally convex functions, the results given here can be proved for locally Lipchitz functions also.
\end{abstract}

Keywords--- Locally Convex Functions, Locally Convex Operators, Uniform Convergence, EPI-Convergence, Strong. Limit Superior, Weak_Limit Inferior

\section{INTRODUCTION}

$\mathrm{T}$ HERE is a lot of study on problems relating to convergence and perturbation of optimization problems. Various modes of convergence have been studied motivated by efforts towards successive approximation schemes in a wide variety of areas including statistics, variational inequalities, approximation theory, convex optimization, control theory and mathematical programming.

Wijsman $[5,6]$ was the first to introduce a new type of convergence, viz., "epigraph convergence in finite dimensions". Uberto Mosco [3] studied the convergence of convex functions using the notion of epigraph convergence in the context of variational problems and convex optimization. Later Bergstorm [2] investigated further the notion of epigraph convergence and applied it to network optimization. Kanniappan and Sundaram Sastry [4] studied the convergence of convex optimization problems under the familiar notion of uniform convergence. Here we study the convergence of locally convex function under uniform convergence in a more general setting. We prove that sequence of locally convex functions converges to a locally convex function.

\section{PRELIMINARIES}

Definition 2.1: Let $\mathrm{X}$ be a locally convex space. A function f: $\mathrm{X} \rightarrow \mathrm{IR}$ is said to be locally convex if (i) the one sided

K. Jeyalakshmi, Department of Mathematics, SRM University, Kattankulathur. E-mail : jayamoor@yahoo.com directional derivative of $f$ at $x_{0}$ in the direction of $x \in X$ is given by

$f^{\prime}\left(x_{o} ; x\right)=\lim _{\lambda \rightarrow 0^{+}} \frac{f\left(x_{0}+\lambda \mathrm{x}\right)-\mathrm{f}\left(x_{o}\right) e}{\lambda}$ exists for all $x \in x$;

(ii) $\mathrm{x} \rightarrow f^{\prime}\left(x_{0} ; x\right)$ is convex and continuous.

If $f$ is locally convex function at all points of $x$, then $f$ is locally convex function on X. [Refer [ ], [ ].A locally convex function is also known as directionally differentiable function in the sense that their directional derivative is convex.

$$
\text { Ex: h: IR } \rightarrow \text { IR by } \mathrm{h}(\mathrm{x})=\log (1+|x|) .
$$

Then $\mathrm{f}$ is locally convex function. However it is not convex. If $\mathrm{f}$ is convex, then it is locally convex function.

\section{LOCALLY CONVEX OPERATORS}

We need the following definitions regarding operators with $\mathrm{X}$ as a locally convex space and $\mathrm{Y}$ as another locally convex space which is also an ordered vector space with the positive cone $\mathrm{C}$.

Let $\mathrm{G}: \mathrm{X} \rightarrow \mathrm{Y}$ be an operator. If the limit $\lim _{\lambda \rightarrow 0^{+}} \frac{G\left(x_{0}+\lambda d\right)-G\left(x_{0}\right)}{\lambda}$ exists, then it is called the one - sided directional derivative of $\mathrm{G}$ at $x_{o}$ in the direction $\mathrm{d}$, and it is denoted by G' $\left(x_{o} ; d\right)$.

It is clear that $\mathrm{G}^{\prime}\left(x_{o} ;.\right)$ is a positively homogenous operator.

An operator $\mathrm{G}: \mathrm{X} \rightarrow \mathrm{Y}$ is said to be locally convex at $x_{0} \in X$, if

1. $\mathrm{G}^{\prime}\left(x_{0} ; x\right)=\lim _{\lambda \rightarrow 0^{+}} \frac{G\left(x_{0}+\lambda_{x}\right)-G\left(x_{o}\right)}{\lambda}$ exists for all $x \in X$, and

2. $\mathrm{x} \rightarrow \mathrm{G}^{\prime}\left(x_{0} ; x\right)$ is continuous and convex.

\section{Definition2.2}

Let $\left\{A_{n}\right\}$ be a sequence of subsets of a locally convex space $X$.

The strong limit inferior of $A_{n}$ denoted by

$\mathrm{s}-\underline{\lim } A_{n}$ is defined by

$\mathrm{s}-\underline{\lim }\left\{a \in X /\right.$ there exists a sequence $a_{n}$ such that $a_{n} \in$ An and $a n \rightarrow a$

Definition 2.3

The strong limit superior of $A_{n}$ denoted by $\mathrm{s}$ $\overline{\lim } A_{n}$ is defined by

$\mathrm{s}-\overline{\operatorname{llm}} \mathrm{A}_{\mathrm{n}}=$

$\left\{a \in X /\right.$ there exists a subsequence $a_{n_{k}}$ such that $a_{n_{k}} \in$ Ank and ank $\rightarrow a$ 


\section{Definition 2.4}

The weak limit inferior of $A_{n}$ denoted by w $-\underline{\lim } A_{n}$ is defined by

$\mathrm{w}-\underline{\lim }$

$A_{n}=\left\{a \in X /\right.$ there exists a sequence $a_{n}$ such that $a_{n} \in$ An and an $\rightarrow$ a weakly

\section{Definition 2.5}

A sequence $A_{n}$ of sets converges to a set $\mathrm{A}$ if and only if $\mathrm{s}-\overline{\operatorname{llm}} \mathrm{A}_{\mathrm{n}}=\mathrm{s}-\underline{\lim } \mathrm{A}_{\mathrm{n}}=\mathrm{A}$.

\section{Definition 2.6}

A sequence $\left\{\mathrm{f}_{\mathrm{n}}\right\}$ of functions is said to converge to a function $f$ epigraphically if and only if the sequence $\left\{\right.$ epi $\left.f_{n}\right\}$ of epigraphs of $\mathrm{f}_{\mathrm{n}^{\prime} \mathrm{s}}$ converges to $\{$ epif\} of epigraph of $f$ in the sense of the definition 2.1

The following equivalent characterization of epi convergence of a sequence of functions can be seen [1].

\section{Definition 2.7}

Let $\mathrm{X}$ be a metrizable locally convex space. A sequence $\left\{\mathrm{f}_{\mathrm{n}}\right\}$ of functions converges to a function $f$ epigraphically at a point $x \in x$ if and only if

(a) There exists a sequence $\mathrm{x}_{\mathrm{n}} \rightarrow \mathrm{x}$ such that

$$
\begin{gathered}
f(x) \geq \lim _{n \rightarrow \infty} \sup f_{n}\left(x_{n}\right) \\
\text { (b) For every } \quad \text { sequence } \quad \mathrm{x}_{\mathrm{n}} \quad \rightarrow \quad \mathrm{x} \text {, } \\
f(x) \leq \lim _{n \rightarrow \infty} \text { inf } f_{n}\left(x_{n}\right)
\end{gathered}
$$

\section{Definition 2.8}

If $\mathrm{X}$ is not metrizable (which is the case when working with weak topologies on Banach spaces), it will be useful to consider the following sequential epiconvergence notions:

$F$ n converges to $f$ epigraphically at $x \in X$ if inf

$$
\left.\liminf f_{n}\left(x_{n}\right) / x_{n} \rightarrow x\right\}=\inf \left\{\lim \sup f_{n}\left(x_{n}\right) / x_{n} \rightarrow x\right\}=f(x) \text { and }
$$

\section{CONVERGENCE THEOREM}

Let $\mathrm{X}$ and $\mathrm{Y}$ be locally convex spaces and let $\mathrm{Y}$ be also an ordered vector space with a normal order cone C. Let $f: \mathrm{X}$ $\rightarrow \mathrm{Y}$ be a continuous locally convex map.

We consider the optimization problem

$$
\inf _{x \in X} f(x)=\alpha
$$

Let $\mathrm{S}=x \in X / f(x)=\alpha$. It may not be easy to solve problem (P). We appropriate $f$ by a sequence $\left\{\mathrm{f}_{\mathrm{n}}\right\}$ of continuous locally convex maps from $\mathrm{X}$ to $\mathrm{Y}$.

Corresponding to each $f_{n}$, we formulate the optimization problem,

$$
\left(\mathrm{P}_{\mathrm{n}}\right) \underset{x \in X}{\inf } f_{n}(x)=\alpha_{n}
$$

Let $\mathrm{S}_{\mathrm{n}}=x \in X / \mathrm{f}_{\mathrm{n}}(\mathrm{x})=\alpha_{n}$
We shall assume that $\mathrm{S}, \mathrm{S}_{\mathrm{n}}, \mathrm{n}=1,2, \ldots$ are non empty subsets of $X$.

By a proper choice of the $f_{n}$ 's it may be easy to solve the problem $\left(\mathrm{P}_{\mathrm{n}}\right)$.

\section{Definition 3.1}

Any sequence $\left\{\mathrm{x}_{\mathrm{n}}\right\}$ such that $x_{n} \in S_{n}$ for $n=$ $1,2, \ldots$ will be called a sequence of solutions of the family $\left(\mathrm{P}_{\mathrm{n}}\right)$.

Definition 3.2 (P) if

The sequence of problems $\left(\mathrm{P}_{\mathrm{n}}\right)$ will be said to converge to

(i) $\quad \alpha_{n} \rightarrow \alpha$;

(ii) If $\mathrm{x}_{\mathrm{n}}$ is a sequence of solutions of $\left(\mathrm{P}_{\mathrm{n}}\right)$ converging to $\mathrm{x} \in \mathrm{X}$, then $\mathrm{x} \in \mathrm{S}$.i.e.s $-\underline{\lim } S_{n} \underline{\mathrm{c}} \mathrm{S}$.

\section{Theorem 3.1}

Let $f_{\mathrm{n}} \rightarrow f$ uniformly. Then the sequence of problems, $\left(\mathrm{P}_{\mathrm{n}}\right)$ converges to problem $(\mathrm{P})$.

Proof:

1. To prove $\alpha_{n} \rightarrow \alpha$

Let $x_{n} \in S_{n}$ and $x_{0} \in S$. Then $f_{\mathrm{n}}\left(\mathrm{x}_{\mathrm{n}}\right)=\alpha_{n}$ and $f\left(x_{0}\right)=\alpha$ Since $f_{\mathrm{n}} \rightarrow f$ uniformly, given any neighborhood $\mathrm{V}$ of the origin in $\mathrm{Y}$, there is an $n_{o} \in N$ such that $f_{n}(x)-f(x) \in$ $V$ for all $n \geq n_{o}$ and for all $x \in X$.

Then we have,

$f_{n}\left(x_{o}\right)-f\left(x_{o}\right) \in V$ for all $n \geq n_{o}$

And

$f_{n}\left(x_{n}\right)-f\left(x_{n}\right) \in V$ for all $n \geq n_{o}$

That is,

$f_{n}\left(x_{o}\right)-\alpha \in V$ for all $n \geq n_{o}$

Also,

$\alpha_{n}-f\left(x_{n}\right) \leq \alpha_{n}-\alpha \leq f_{n}\left(x_{o}\right)-\alpha$

Since $\mathrm{Y}$ is normally ordered, it follows from (2) to (3) and (4) that $\alpha_{n}-\alpha \in V$ for all $\mathrm{n} \geq \mathrm{n}_{\mathrm{o}}$.

That is, $\alpha_{n}-\alpha$

i. Let $\left\{x_{n}\right\}$ be a sequence of solutions of the problems $\left(\mathrm{P}_{\mathrm{n}}\right)$ such that $x_{n} \rightarrow x_{o}$.

To prove $x_{o} \in S$

Let $\mathrm{V}$ be a full symmetric neighborhood of the origin in $\mathrm{Y}$. Since $f$ is continuous, we have

$\mathrm{f}\left(\mathrm{x}_{\mathrm{n}}\right) \rightarrow \mathrm{f}\left(x_{0}\right)$.

Hence there is an $\mathrm{n}_{1} \in N$ such that $f\left(x_{n}\right)-f\left(x_{o}\right) \in$ 
$\frac{1}{3} V$ for all $n \geq n_{1}$

Hence there is an $\mathrm{n}_{2} \in N$ such that

$\alpha_{n}-\alpha \in \frac{1}{3} V$ for all $n \geq n_{2}$

Since $f_{n}(x) \rightarrow f$ uniformly, there is an $\mathrm{n}_{3} \in N$ such that $f_{n}(x)-f(x) \in \frac{1}{3} V$ for all $n \geq n_{3}$ and for all $x \in \mathrm{X}(7)$

Let $n_{o}=\max \left\{n_{1}, n_{2}, n_{3}\right\}$

From (7), we have

$$
f_{n_{o}}\left(x_{n_{o}}\right)-f\left(x_{n_{o}}\right) \in \frac{1}{3} V
$$

That is,

$\alpha_{n_{o}}-f\left(x_{n_{o}}\right) \in \frac{1}{3} V$

From (6)

$\alpha_{n_{o}}-\alpha \in \frac{1}{3} V$

or

$$
\alpha-\alpha_{n_{o}} \in \frac{1}{3} V
$$

From (5), we have

$\mathrm{f}\left(x_{n_{o}}\right)-f\left(x_{o}\right) \in \frac{1}{3} V$

From (8), (9) and (10) we have,

$\alpha-f\left(x_{o}\right) \in V$

Since $\mathrm{V}$ is an arbitrary neighborhood of zero in $\mathrm{Y}$ we have $x_{o} \in \mathrm{S}$.

That is, $\mathrm{s}-\underline{\lim } S_{n} \underline{\mathrm{c}} \mathrm{S}$.

From (i) and (ii), we have $\left(\mathrm{P}_{\mathrm{n}}\right) \rightarrow(\mathrm{P})$

Corollary 3.1

If $\cup S_{n}$ is relatively compact, then every sequence of solutions of $\left(\mathrm{P}_{\mathrm{n}}\right)$ has a subsequence which converges to a solution of $(\mathrm{P})$.

That is, $\mathrm{s}-\overline{l \iota m} S_{n} \subseteq S$

The question that naturally arises is whether, $\mathrm{s}-\overline{l \iota m} S_{n} \subseteq$ $S_{n}$, is in fact equal to $S$. In other words, if $x_{o}$ is a solution of $(\mathrm{P})$, is it necessarily the limit of a sequence of solutions of $\left(\mathrm{P}_{\mathrm{n}}\right)$ ? That such is not the case generally can be seen from the following example.

Consider the following real valued functions defined on the real line.

$$
f(x)=\left\{\begin{array}{cc}
x^{2} & \text { for }|x|>1 \\
1 & \text { for }|x| \leq 1
\end{array}\right.
$$

$$
f_{n}(x)=\left\{\begin{array}{cc}
x^{2} & \text { for }|x|>1 \\
1-\frac{1}{n}(x+1) & \text { for }-1 \leq x \leq 0 \\
1+\frac{1}{n}(x-1) & \text { for } 0 \leq x \leq 1
\end{array}\right.
$$

It is easy to see that $\left\{f_{n}\right\}$ is a sequence of continuous convex functions (hence locally convex) converging uniformly to the continuous convex function $f$. It is also clear that

$\mathrm{S}_{\mathrm{n}}=\{0\}$ for $\mathrm{n}=1,2, \ldots \quad$ But $\mathrm{S}=[-1,1]$.

Thus, $\mathrm{s}-\overline{l \imath m} S_{n} \subsetneq \mathrm{S}_{\mathrm{n}}$

It can be easily proved that if $\mathrm{X}$ is a locally convex space and $\mathrm{Y}=\mathrm{R}$ and $\mathrm{f}_{\mathrm{n}}, \mathrm{n}=1,2$, and $f$ are $1-1$ with $\mathrm{f}_{\mathrm{n}} \rightarrow f$ uniformly. Then, $\mathrm{s}-\underline{\lim } S_{n}=\mathrm{S}$

Theorem 3.2 - Epi-Convergence and uniform convergence

Let $\left\{f_{\mathrm{n}}\right\}$ converge to $f$ uniformly. Then $\left\{f_{\mathrm{n}}\right\}$ converges to $f$ epigraphically.

Proof:

Let $x_{o} \in X$ and $x_{n} \in X$ be such that $x_{n} \rightarrow x_{o}, f_{n} \rightarrow f$ uniformly. Given $\in>0$, there exists $\mathrm{n}_{1} \in \mathrm{N}$ such that $\left|f_{n}\left(x_{n}\right)-f(x)\right|<\epsilon / 2$ for all $\mathrm{n} \geq \mathrm{n}_{1}$.

Since $x_{n} \rightarrow x_{o}$ and $f$ is contagious, there exists $\mathrm{n}_{2} \in \mathrm{N}$ such that

$\mid f_{n}\left(x_{n)-f(x)} \mid<\in / 2\right.$ for all $\mathrm{n} \geq \mathrm{n}_{2}$

Let $\mathrm{n} 0=\max \{\mathrm{n} 1, \mathrm{n} 2\}$. (10) and (11) imply, for all $\mathrm{n} \geq \mathrm{n}_{0}$

$\left|f_{n}\left(x_{n}\right)-f\left(x_{0}\right)\right| \leq\left|f_{n}\left(x_{n}\right)-f\left(x_{n}\right)\right|+\mid f_{n}\left(x_{n}\right)-$ $f(x 0)<\epsilon 2+\epsilon 2=\epsilon$

That is $f_{n}\left(x_{n}\right) \rightarrow\left(x_{o}\right)$, whenever $x_{n} \rightarrow x_{o}$. We have $f_{n} \rightarrow f \quad$ epigraphically $\quad$ at $\quad x_{o} \in X \quad$ if Inf $\left\{\overline{\lim } f_{n}\left(x_{0}\right) / x_{n} \rightarrow x_{o}\right\}=\inf \left\{\underline{\lim } f_{n}\left(x_{0}\right) / x_{n} \rightarrow x\right\}=f$ $\left(x_{0}\right)$

(11) and (12) together imply $f_{n} \rightarrow f$ epigraphically at $x_{0}$. Since $x_{0} \in X \quad$ is arbitrary, we have $f_{n} \rightarrow f$ epigraphicallly. Hence the theorem.

\section{CONCLUSION}

We have outlined the effects of general perturbations of the problem functions on solution functions under the notion of the existence of directional derivative in vector optimization. It can be considered for general convex stochastic programming with parameter which is random variables. Given the close connection between solution algorithm and sensitivity calculation, it is obvious that an algorithm can determine a solution, and then algorithmic manipulation can be utilized for sensitivity information. Useful computable techniques can be developed and implemented. Convergence of Lagrange multipliers can be considered. 


\section{REFERENCES}

[1] H. Attouch, "Epi convergence and duality, optimization and related fields", Lecture notes in Mathematics, Vol. 1190, Springer - Vulag, 1984.

[2] R. Bergstorm, "Optimization, convergence and duality", Thesis, University of Illinois at Urbana Champaign, 1980.

[3] U. Mosco, "Convergence of convex sets and of solutions of variational inequalities", Advance in Math. 3, Pp. 510 - 585, 1969.

[4] Kanniappan and Sundharam Sastry, "Uniform convergence of Convex Optimization problem”, J. Math. Anal. Appl. 96, No.1, Pp. 1-12, 1983.

[5] R.A.Wijsman, "Convergence of sequence of convex sets, cones and functions-I”, Bull.Amer.Math.Soc., Vol. 70, Pp. 186-188, 1964.

[6] R.A.Wijsman, "Convergence of sequence of convex sets, cones and functions- II”, Trans.amer.Math.soc.,123, Pp. 32-45, 1966. 